\title{
Design of Certain Diagnostic Expert System for the Aircraft Armament Equipment
}

\author{
Wang yu-gang, Wang heng-xin, Zhang dan-feng, Chen ye \\ Department of aviation armament fire control system, Naval Aeronautical Engineering Academy of \\ Qingdao Branch, Qingdao, 266041, China \\ flyingmanw@163.com
}

Keywords: fault diagnosis; expert system; database

\begin{abstract}
The diagnostic expert system is applied widely in ATS, and it is one of the pivotal technologies in the automatic test and diagnosis field. This paper constructs a diagnostic expert system for the aircraft armament equipment. The expert system build database with LabSQL. It realize data storage、management and querying, and the proposal of condition-based maintenance was brought forward. This method improves fault detection rate and fault diagnosis efficiency.
\end{abstract}

\section{Introduction}

At present, the equipment of military aircraft becomes increasingly large 、 complex 、 networked、 replacement fast、high-tech 、 expensive, and fault covers a wide fields. To protect for troops is difficult. Especially for aviation ordnance specialized field, its support work directly related to weapons, the slightest mistake will lead to disaster, causing heavy casualties and huge property losses. To make up for deficiencies in maintenance fields and fault diagnosis, according to the following three principles, (1) for the use of troops of equipment, maintenance, support personnel system development; (2) comply with the actual fields., friendly interface and operability strong; (3) focus on safety and reliability design. Design and development of a certain type of machine fault diagnosis expert system equipment.

\section{Based on ADO and SQL LabSQL}

\subsection{LabSQL Introduction}

LabSQL is a cross-platform database access toolkit of LabVIEW. It uses Microsoft ADO and SQL language to complete the database access, the complexities of the underlying ADO and SQL operations packaged into a series of LabSQL VI, by its very nature is a series encapsulates the ADO VI set of properties and methods. LabSQL support for any Windows operating system-based ODBC databases, including Access, SQL Server, Oracle, Sybase, etc.

Advantages LabSQL is easy to understand, easy to operate, are not familiar with the SQL language users can easily use. Simply programming, database access can be achieved in LabVIEW, perform various SQL queries, and perform various operations on record. Using LabSQL can access any type of database, recording the various operations. Another advantage is its biggest source code is open, and is completely free.

\subsection{LabSQL configuration}

LabSQL through ODBC connection between the database, the user needs to specify the data source name and driver in ODBC. Therefore, before using LabSQL, you first need a Windows operating system ODBC data source to create a DSN (data source name, data source name). LabSQL connection between the database is built on the basis of DSN. Connected flow shown in Figure 1. 


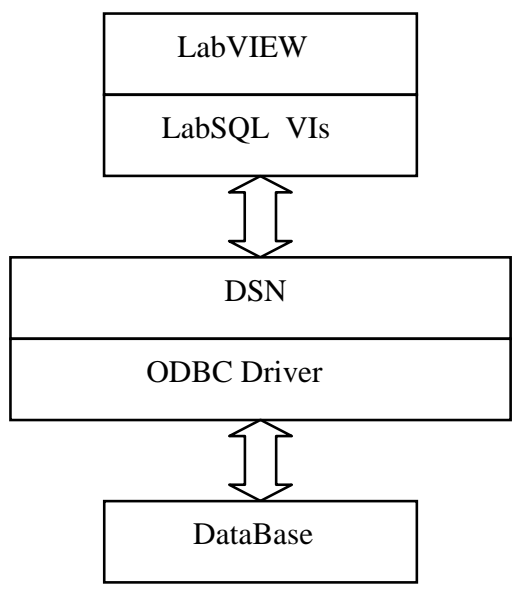

Figure 1 diagram of LabSQL connected with database

\section{Study of fault Rules}

A certain type of aircraft equipment in the work process, found fault there are three main ways: visual, manual inspection, various BIT testing. Where all faults are detected and fault are saved in the maintenance list (MFL) in. and fault maintenance list, to indicate a fault with and fault code information, a typical format of a failure code is displayed as shown in Figure 2.

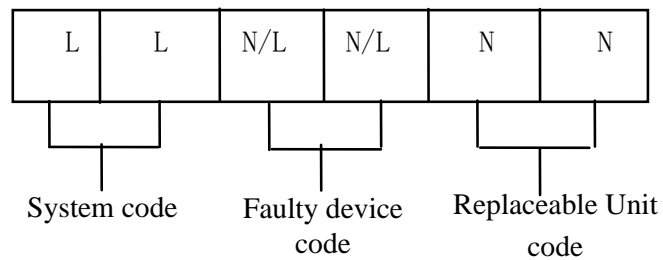

Figure 2 Fault code display format

A total of six fault codes, the figure represents the letter $\mathrm{L}, \mathrm{N}$ represents a number. 1,2 indicates a system failure code, 3,4 indicates a faulty device code, that is, line replaceable unit (LRU) code that represents the fifth and sixth place in the field replaceable unit (SRU) and function code. According to the fault code can determine the corresponding location of the fault.

First, maintained in accordance with expertise and field experience of the staff, get and fault and fault diagnosis knowledge of the equipment, based on the failure analysis, to establish fault rule a certain type of aircraft equipment, and the fault rule is expressed as follows:

Rule（NUM,FORE,NAME,FITM,DETM,TIMES,ISO,SOL）

Namely: fault rules (number, the higher the number, the fault code or name, the first time and fault, the fault duration occurs, the number of and fault isolation, troubleshooting measures to happen).

The following equipment, for example, to analyze the situation of each LRU and SRU might malfunction, shown in Figure 3

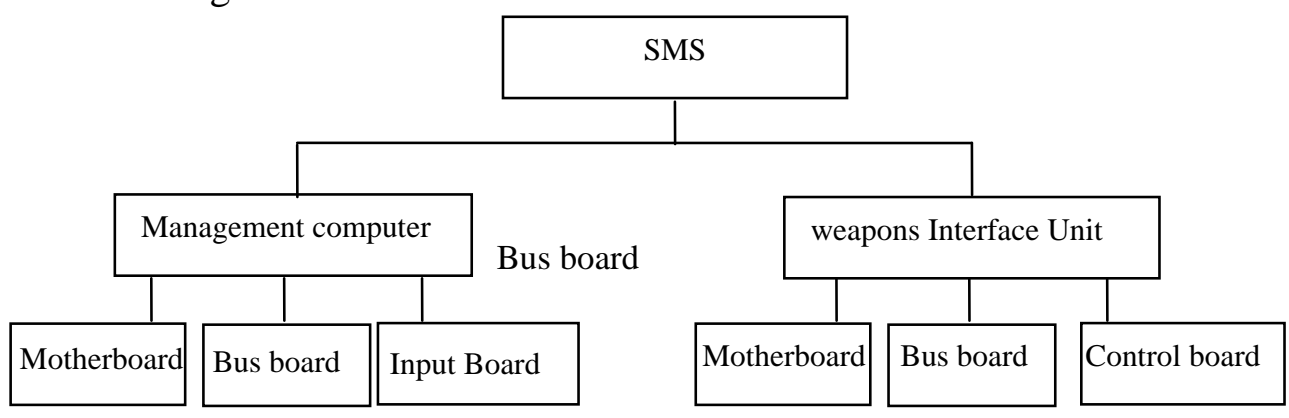

Figure 3 system fault level example of Figure 


\section{Design of Fault Diagnosis Expert System}

Schematic certain type of aircraft equipment fault diagnosis expert system shown in Figure 4. It consists of several parts troubleshooting rule base, diagnostic reasoning module, explaining agencies, diagnostic knowledge maintenance modules. Among them, the fault diagnosis rule base and knowledge base management module is used to store and manage diagnostic knowledge, you can achieve read the rules, deletions and modifications. The contents of the database nodes from different test machine, field replaceable unit LRU, SRU within the field replaceable unit to each functional module block storage, this design can improve the efficiency of the expert system inference.

\subsection{Database}

In a certain type of aircraft equipment fault diagnosis expert system knowledge comes from two aspects: the literature of this type of devices, including technical specifications, failure mode analysis, manual, commissioning records and common faults and troubleshooting methods; experts in the field and equipment maintenance experience personnel, including knowledge of the structure of the system maintenance process, causal knowledge and behavior knowledge. The database includes: the rule base and case base. The knowledge base can be transformed between each other to accommodate different types of faults. In this paper, Access creates a database called " fault", the database contains a certain type of aircraft equipment failure.

\subsection{Inference Engine}

Carrying out knowledge representation, the relevant information for each node contains and fault tree species troubleshooting steps. For each fault tree table top event fault tree analysis corresponds to the expert system is to solve the task, which corresponds to the end of the event the expert system inference result, the corresponding fault tree from the top in the end of the level and logic reasoning in expert system . In this system, through the rules for and fault analysis, coupled with a direct subordinate faults in the fault information stored in ID, you can achieve and fault tree traversal search for organizational structure. In the troubleshooting process, the need to establish a definite correspondence between the storage location of the fault $f$ record and its keywords so that each keyword and the results are stored in a unique position, respectively. Thus to find the value of $\mathrm{K}$ is given as $\mathrm{f}(\mathrm{K})$ according to the corresponding relationship $\mathrm{f}$. According to this thinking to establish a hash table. Program hash table and fault information stored query diagram shown in Figure 4:

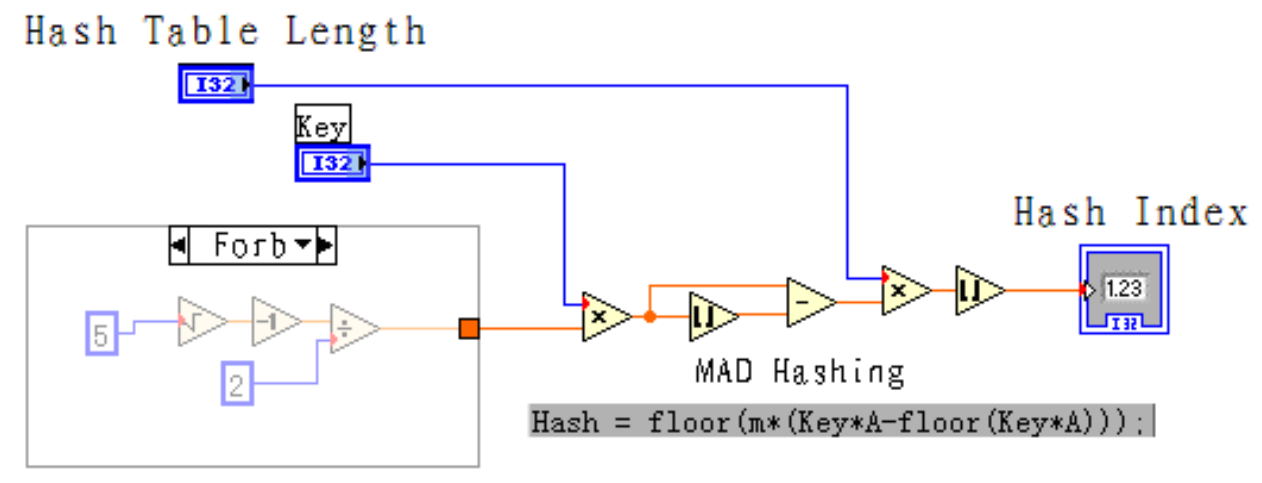

Figure 4 Inference engine block diagram

\section{3 explain the mechanism}

When a user seeks to explain, the system will make the appropriate node back, starting from the user originally selected phenomena search every step of the reasoning process that is relevant information for each node, and then remove the node corresponding to the relevant information from the fault database , they are displayed on the interpretation of the interface. For the equipment fault diagnosis system, through the and fault of the judge, given the measures fault isolation and corrective measures, and through failure analysis closer to identify and fault produced a variety of reasons, in order to achieve devices for intelligent diagnostics and maintenance . 


\section{Conclusion}

Certain type of machine fault diagnosis expert system software to run user-friendly, easy to operate. Effectively improve the detection rate and diagnostic troubleshooting efficiency of diagnostic systems. Realized by constructing the recording and fault location fault tree, exclude verify preliminary results achieved comprehensive diagnosis. Because of the accumulation of knowledge and information is the basis for obtaining diagnostic reasoning and diagnostic algorithms, expert systems therefore need further verification and improvement of the knowledge base needed for timely optimization and additions.

\section{References:}

[1] Li Jian-wen, Liu Du-xi, Zhu Ming-quan, the method ADO technology to access the database LabVIEW [J] Automation Instrumentation, 2003,24 (9): p15-17

[2] Xu Hong-an, Fei Ren-yuan, Wang Min, constructed in LabVIEW with ADO database access interface [J], Beijing University of Technology, 2003,29 (2): p138-140

[3] Yang Le-ping, Li Hai-tao, Zhao Yong, LabVIEW Advanced Programming, April 2003, Tsinghua University Press, p470-485

[4] Liu Yong, navigation fault diagnosis expert system based on fault tree [J] Naval University of Engineering report, 2002, (1):. P41-44

[5] Yang Jun, Feng Zhen-sheng, Huang Kao-li, equipment intelligent fault diagnosis technology [M] Beijing: National Defense Industry Press, 2004: p84- 86 